\title{
NRF2-driven miR-125B1 and miR-29B1 transcriptional regulation controls a novel anti-apoptotic miRNA regulatory network for AML survival
}

\author{
NM Shah ${ }^{1,2}$, L Zaitseva ${ }^{1}$, KM Bowles $^{1,3}$, DJ MacEwan ${ }^{2,4}$ and SA Rushworth ${ }^{*, 1,4}$
}

Transcription factor NRF2 is an important regulator of oxidative stress. It is involved in cancer progression, and has abnormal constitutive expression in acute myeloid leukaemia (AML). Posttranscriptional regulation by microRNAs (miRNAs) can affect the malignant phenotype of AML cells. In this study, we identified and characterised NRF2-regulated miRNAs in AML. An miRNA array identified miRNA expression level changes in response to NRF2 knockdown in AML cells. Further analysis of miRNAs concomitantly regulated by knockdown of the NRF2 inhibitor KEAP1 revealed the major candidate NRF2-mediated miRNAs in AML. We identified miR-125B to be upregulated and miR-29B to be downregulated by NRF2 in AML. Subsequent bioinformatic analysis identified putative NRF2 binding sites upstream of the miR-125B1 coding region and downstream of the mir-29B1 coding region. Chromatin immunoprecipitation analyses showed that NRF2 binds to these antioxidant response elements (AREs) located in the $5^{\prime}$ untranslated regions of miR-125B and miR-29B. Finally, primary AML samples transfected with anti-miR-125B antagomiR or miR-29B mimic showed increased cell death responsiveness either alone or co-treated with standard AML chemotherapy. In summary, we find that NRF2 regulation of miR-125B and miR-29B acts to promote leukaemic cell survival, and their manipulation enhances AML responsiveness towards cytotoxic chemotherapeutics.

Cell Death and Differentiation (2015) 22, 654-664; doi:10.1038/cdd.2014.152; published online 17 October 2014

Acute myeloid leukaemia (AML) is a biologically heterogenous disorder that occurs as a consequence of a wide variety of genetic abnormalities in haematopoietic progenitors that are derived from the bone marrow. ${ }^{1}$ However, it is likely that $A M L$ share various survival pathways downstream of the driver mutations, making the existence of common survival pathways and therapeutic targets likely. ${ }^{2}$

Nuclear factor (erythroid-derived 2)-like 2 (NRF2) is a member of the Cap ' $n$ ' Collar basic leucine zipper transcription factor family that protects cells from reactive oxygen species through the regulation of a number of cytoprotective genes including heme oxygenase-1 $(\mathrm{HO}-1)$ and $\mathrm{NAD}(\mathrm{P}) \mathrm{H}$ dehydrogenase quinone 1 (NQO1). ${ }^{3-5}$ In cancer, NRF2 activation is pro-tumoural in a spectrum of malignancies through mutations in NRF2 or its cytosolic inhibitor KEAP1.$^{6-9}$ However, we have previously shown that in human AML constitutive activation of the NRF2 signalling pathway is not through somatic mutations of NRF2/Keap1 but as a consequence of upstream constitutive activation by NF-kB. ${ }^{10,11}$ In addition to contributing to the malignant phenotype of AML, we also found that NRF2 contributes to intrinsic leukaemia cell resistance to standard front-line chemotherapy agents. ${ }^{12,10}$

microRNAs (miRNAs) are short non-coding RNA molecules, approximately 20-30 nucleotides in length, that can posttranscriptionally regulate gene expression by binding to the $3^{\prime}$ UTR of their target mRNA, thereby repressing gene transcription. ${ }^{13}$ miRNA expression has shown to be dysregulated in a range of cancers, and can act as tumour suppressor miRs (by downregulating oncogenes) or oncomiRs (by targeting tumour suppressors). ${ }^{14}$ miRNAs are being increasingly studied with the expectation that modulation of their expression can be harnessed to improve cancer therapy. A recent study using chromatin immunoprecipitation sequencing (ChIP-Seq) identified high-confidence ChIP-Seq peaks for NRF2 binding in the vicinity of several miRNAs, suggesting they could be regulated by NRF2. ${ }^{15}$

In the present study, we look to identify whether NRF2 regulates miRNA in human AML. Furthermore, we evaluate whether NRF2-regulated miRNAs have functional importance not only on the biology of the disease but also in the leukaemia cell death to chemotherapy treatment.

\section{Results}

Lentiviral NRF2 knockdown alters miRNA expression. NRF2 can regulate drug resistance in AML via the induction of cytoprotective and detoxification genes. ${ }^{10,12}$ To assess whether NRF2 regulates miRNA expression in AML, we used

\footnotetext{
${ }_{1}^{1}$ Department of Molecular Haematology, Norwich Medical School, University of East Anglia, Norwich Research Park, Norwich, UK; ${ }^{2}$ Department of Molecular and Clinical Pharmacology, Institute of Translational Medicine, University of Liverpool, Liverpool, UK and ${ }^{3}$ Department of Haematology, Norfolk and Norwich University Hospitals NHS Trust, Colney Lane, Norwich, UK

${ }^{*}$ Corresponding author: SA Rushworth, Department of Molecular Haematology, Norwich Medical School, Norwich Research Park, Norwich, NR4 7TJ, UK. Tel: +44 (0)1603 591802; Fax: +44 (0)1603 591750; E-mail: s.rushworth@uea.ac.uk

${ }^{4}$ These authors contributed equally to this work.

Abbreviations: AML, Acute myeloid leukaemia; NRF2, Nuclear factor (erythroid-derived 2)-like 2; HO-1, heme oxygenase-1; NQO1, NAD(P)H dehydrogenase quinone 1; NRF2-KD, NRF2 lentiviral knockdown; NEG-KD, negative control knockdown; ChIP, chromatin immunoprecipitation assay; miRNAs, microRNAs

Received 25.2.14; revised 23.8.14; accepted 25.8.14; Edited by A Villunger; published online 17.10.14
} 
a commercially available OncomiR Cancer miRNA qRT-PCR Array on THP-1 cells infected with an NRF2 lentiviral knockdown (NRF2-KD) and a negative control knockdown (NEG-KD). Figure 1a shows the change in expression of miRNA in response to NRF2-KD compared with the control infected with NEG-KD. We then treated THP-1 cells with the NRF2 activator sulforaphane for $6 \mathrm{~h}$ for comparison. Supplementary Table 1 shows the fold increase of miRNA in response to both NRF-KD and sulforaphane treatment. From these experiments, we identified miR-125B, miR-222, miR-221 and miR-223 as potential miRNAs upregulated by NRF2 and miR-29B and miR-154 as potential miRNAs downregulated by NRF2. Figure 1c shows western blot analysis of NRF2 and KEAP1 protein expression in THP-1 cells in response to NRF2-KD, KEAP1-KD and NEG-KD.

Increased NRF2 activity increases miR-125B and decreases miR-29B expression in AML. We used a qRTPCR approach to validate the data from the lentiviral array experiments. ${ }^{10}$ NRF2-KD results in THP-1 cells showing a consistent decrease in miR-125B expression and increasing miR-29B expression as well as smaller but not significant fluctuations in miR-221, miR-222, miR-223 and miR-154 expression (Figure 1b). To further assess the role of NRF2 in regulating miRNA, we increased the activity of NRF2 in THP-1 cells by knockdown of the NRF2 inhibitor KEAP1. ${ }^{16}$ THP-1 were infected with KEAP1 lentiviral knockdown (KEAP1-KD). QRT-PCR confirmed the downregulation of KEAP1 and as a positive control, we showed that KEAP-KD upregulated the NRF2 target gene HO-1. Moreover, miR-125B expression showed a similar increase to $\mathrm{HO}-1$ and miR-29B showed a significant decrease in response to KEAP1-KD (Figure 1b). The expression of miR-221, miR-222, miR223 and miR-154 were not significantly changed in response in NRF2-KD or KEAP1-KD to warrant its inclusion in further experiments.
NRF2 regulates homologues $\mathrm{miR}-125 \mathrm{~B} 1$ and $\mathrm{miR}-29 \mathrm{~B} 1$ and miR-29A in human AML. Both miR-125B and miR-29B exist as two homologues (Figure 2a). miR-125B1 (located on chromosome 11) and miR-125B2 (located on chromosome 21 ), both contain identical seed sequences. Both homologues lie in the region of regulatory miRNA clusters, miR-100-let-7-a-2 and LINC00478, respectively. miR-29B also exists as two homologues, miR-29B1 (located on chromosome 7) and miR-29B2 (located on chromosome 1) (Figure 2a).

The sequence of the miRNA seed region of both miR-125B1 and 2 is identical. Similarly, the seed sequence of miR-29B1 is identical to that of miR-29B2. Therefore, we used primers designed specifically for the immature miRNA sequences (which does differ between the miRNA homologues) to identify which miR-125B and miR-29B homologues were being regulated by NRF2. QRT-PCR on lentiviral NRF2-KD THP-1 cells identified reduced expression of the immature miR-125B1 and increased expression of the immature miR-29B1, but no change in the expression of immature $\mathrm{miR}-125 \mathrm{~B} 2$ and miR-29B2 sequence (Figures $2 \mathrm{~b}$ and $c$ ). This suggests that miR-125B1 and miR-29B1, but not miR-125B2 or miR-29B2, are regulated by NRF2.

To determine whether NRF2 specifically regulated miR-125B1 and miR-29B1 or had a more general regulatory effect on their clusters, we looked for a differential expression on other miRNAs in each of the homologue regions in response to NRF2-KD. Following NRF2 knockdown, however, there was no difference in the expression of miR-100 and let-7a-2 (miR-125B1 cluster; chromosome 11) or miR-99a and let7c (miR-125B2 cluster; chromosome 21) (Figure 2b). This implies that NRF2 does not regulate these clusters to control miR-125B expression but instead binds to a miR-125B1specific regulatory region. Conversely, knockdown of NRF2 elevated miR-29B1 and miR-29A but had no effect on miR-29B2 and miR-29C (Figure 2c). NRF2 therefore regulates
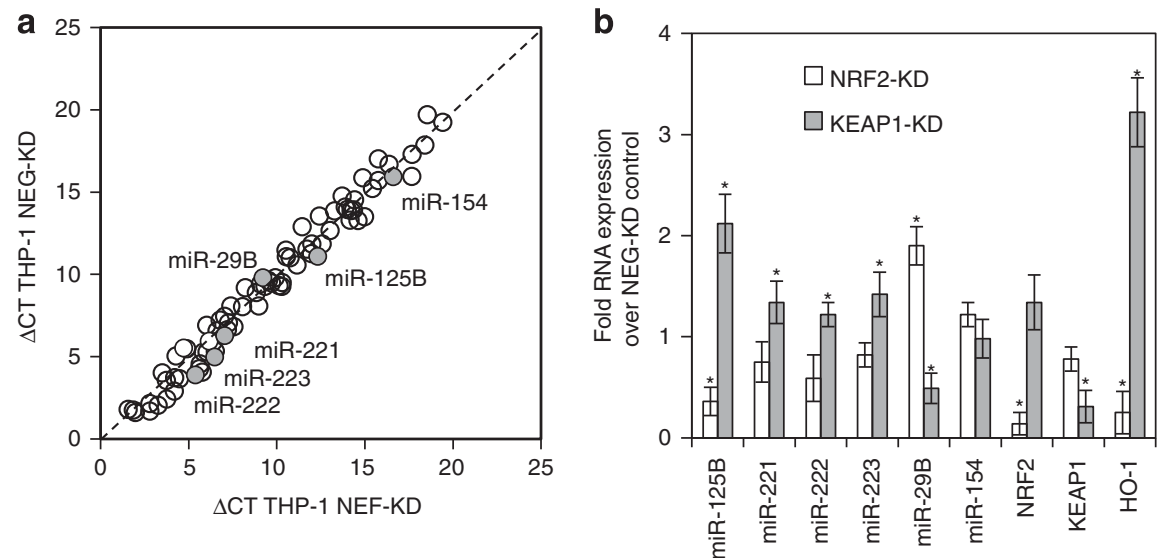

C

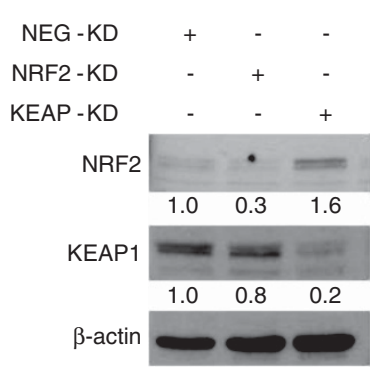

Figure 1 miRNA profiling of AML cells in response lentiviral NRF2 knockdown. (a) THP-1 cells were transduced with NEG (NEG-KD)- and NRF2 (NRF2-KD)-targeted miRNA lentiviral constructs. QRT-PCR analysis of 92 cancer-associated miRNAs in NRF2-KD THP-1 cells. Values represent change in qRT-PCR cycle threshold normalised to RNU6B $(\Delta C T)$. Dashed line indicates no change in expression. A red circle indicates miR-125B, miR221, miR-223, miR222, miR29B and miR154. (b) QRT-PCR of miR-125B, miR221, miR-223, miR222, miR29B, miR154, NRF2, KEAP1 and HO1 in THP-1 cells transduced with NEG-KD, NRF2-KD or KEAP1-KD. Values represent fold change in RNA expression over NEG-KD control. (c) THP-1 were transduced with NEG-KD, NRF2-KD or KEAP1-KD before cells were analysed for NRF2 and KEAP1 using western blotting. Blots were reprobed for $\beta$-actin to show sample loading. The numbers under the blots indicate densitometry analysis of the blots using Image $\mathrm{J}$ software, and the results are expressed as fold change relative to the NEG-KD control 
a

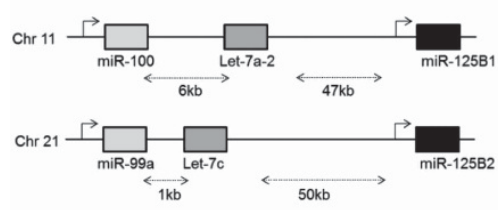

b

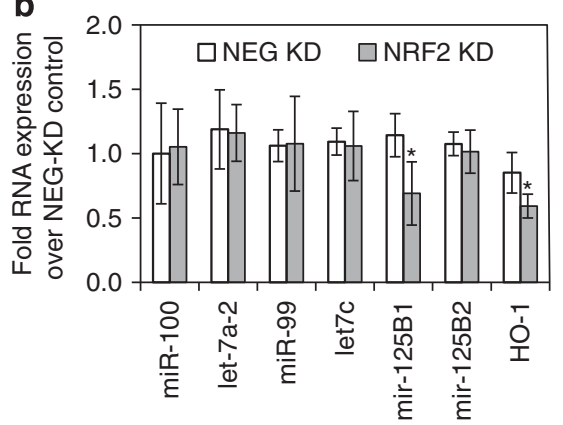

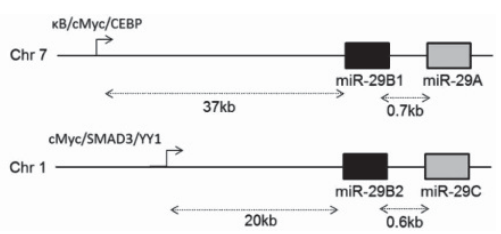

C

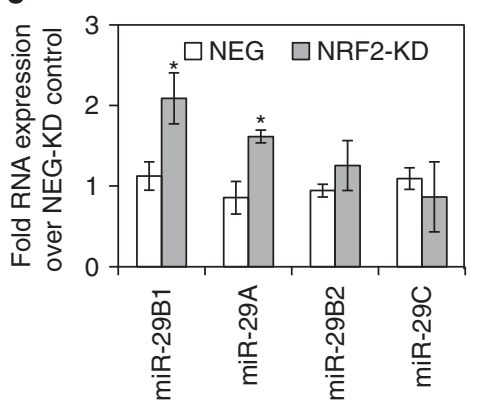

Figure 2 NRF2 regulates homologues miR-125B1 and miR-29B1 and miR-29A in human AML. (a) Schematic representation of the miRNA chromosomal positioning of miR-125B (1 and 2) and miR-29B (1 and 2). (b) Total RNA was extracted from THP-1 transduced with NEG-KD and NRF2-KD and examined for miRNA expression including immature miR-125B1, miR-125B2 RNA and HO-1 mRNA expression. (c) Total RNA was extracted from THP-1 transduced with NEG-KD and NRF2-KD and examined for miR-29A and miR-29C expression including immature miR-29B1, miR-29B2 RNA and HO-1 mRNA expression

a defined pattern of expression of the miR-125B1 and miR-29B1 cluster in human AML. Moreover, results from Figure $1 \mathrm{~b}$ and Figures $2 \mathrm{~b}$ and $\mathrm{c}$ show that changes in total levels of miR-125B and miR-29B are similar to changes in miR-125B1 and miR-29B1. The main reason for this similarity is that constitutive levels of miR-125B2 and miR-29B2 are much lower in $A M L$ than that of their homologue, thus changes observed in total miR-125B and miR-29B reflect changes observed in miR-125B1 and miR-29B1 levels.

NRF2 binds to ARE sites in the promoters of miR-125B1 and miR-29B1. NRF2 acts as a transcription factor by binding to the antioxidant response element (ARE) region in the promoter regions of its target genes. ${ }^{17}$ To analyse the 5' miR-125B1 and miR-29B1 promoter sequence, we used transcription factor analysis programmes (http://www.generegulation.com/cgi-bin/pub/programs/pmatch/bin/p-match.cgi and www.genomatix.de/matinspector.html). These analyses identified three potential ARE sites within the miR-125B1 promoter and two in the miR-29B1 promoter (Figure 3a).

To confirm whether NRF2 bound to ARE binding sites in the miR-29B1 or the miR-125B1 promoter, a ChIP assay was undertaken. Figure $3 \mathrm{~b}$ shows the analysis using antibodies for NRF2 and quantified against control IgG with specific primers for ARE1-3 (promoter region of miR-125B1; chromosome 11) and ARE4 and ARE5 (promoter region of miR-29B1; chromosome 7). Recruitment of NRF2 was markedly enhanced to ARE5 in the miR-29B1 promoter and ARE3 in the miR-125B1 promoter. Next, we evaluated the recruitment of NRF2 to ARE1-5 sites in THP-1 cells transfected with KEAP1 siRNA. Figure $3 c$ shows that there was increased recruitment of NRF2 to the ARE3 and ARE5 site, but not the other potential ARE sites in THP-1 cells transfected with KEAP siRNA over control SiRNA. These observations confirmed that NRF2 specifically binds the miR-29B1 ARE5 site and the miR-125B1 ARE3 site.
To establish whether NRF2 functionally controls miR-125B1 expression, the miR-125B1 promoter was cloned into a PGL4 luciferase plasmid and the putative ARE3 site was mutated (Figure $3 d$ ). AML is a disease characterised by constitutive activation of NRF2, therefore the luciferase vector PGL4 and PGL4/p125B were transfected into THP-1 cells without prior treatment of an NRF2 activator or repressor. We observed an approximate eightfold increase in luciferase activity in the PGL4/p125B plasmid in comparison with the control (Figure $3 e$ ). Site-directed mutagenesis of the ARE3 site in the miR-125B1 promoter corresponded with a significant decrease in miR-125B1 promoter activity (Figure 3e). Moreover, when we co-transfected KEAP1 and NRF2 siRNA with the $\mathrm{p} 125 \mathrm{~B}$ and $\mathrm{p} 125 \mathrm{BNRF}$ Mut plasmids, KEAP1 siRNA induced significant increase in p125B promoter activity and NRF2 siRNA inhibited p125B promoter activity. KEAP1 siRNA and NRF2 siRNA had no effect on p125BNRF2Mut promoter activity (Figure 3f). Similar experiments on the miR-29B1 promoter were technically not possible as the construct was too big and would not clone into a luciferase reporter vector. These results demonstrate that the ARE3 site in the promoter of mir-125B1 is regulated by NRF2.

NRF2 regulates the expression of miR-125B1 and miR-29B1 in primary human AML. As we have established in $\mathrm{AML}$ that high NRF2 mRNA expression causes an increase in NRF2 activity, ${ }^{10}$ and that miR-125B1 and miR-29B1 are regulated by NRF2 in AML cell lines, we next examined the expression of NRF2, mir-125B1 and miR-29B1 in primary human AML cells. NRF2 and miR-125B1 is increased in AML compared with normal CD34+ haematopoietic stem cells (HSC), whereas miR-29B1 is decreased in AML compared with normal CD34+ HSC (Figure 4a). As total levels of miR-125B and miR-29B most likely determine their biological function, we analysed total levels of miR-125B and 
a

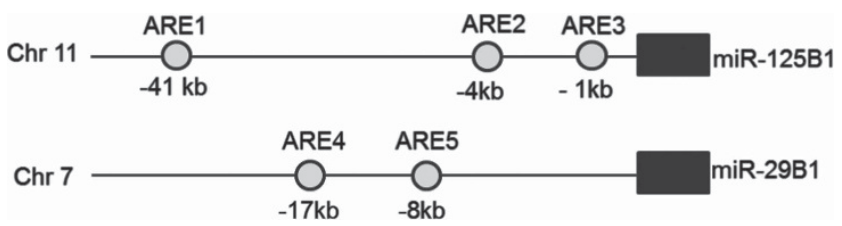

b

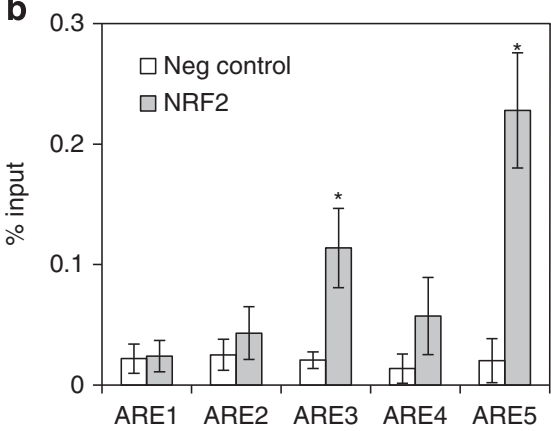

C

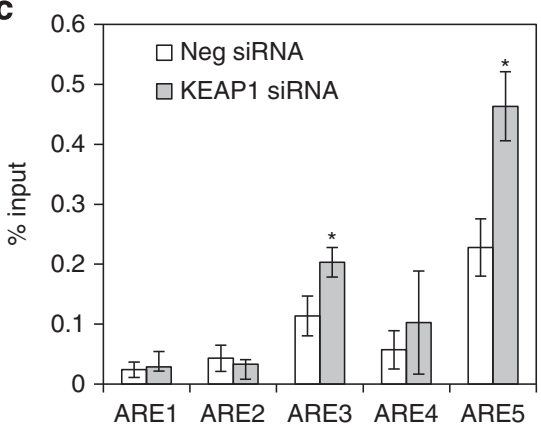

d
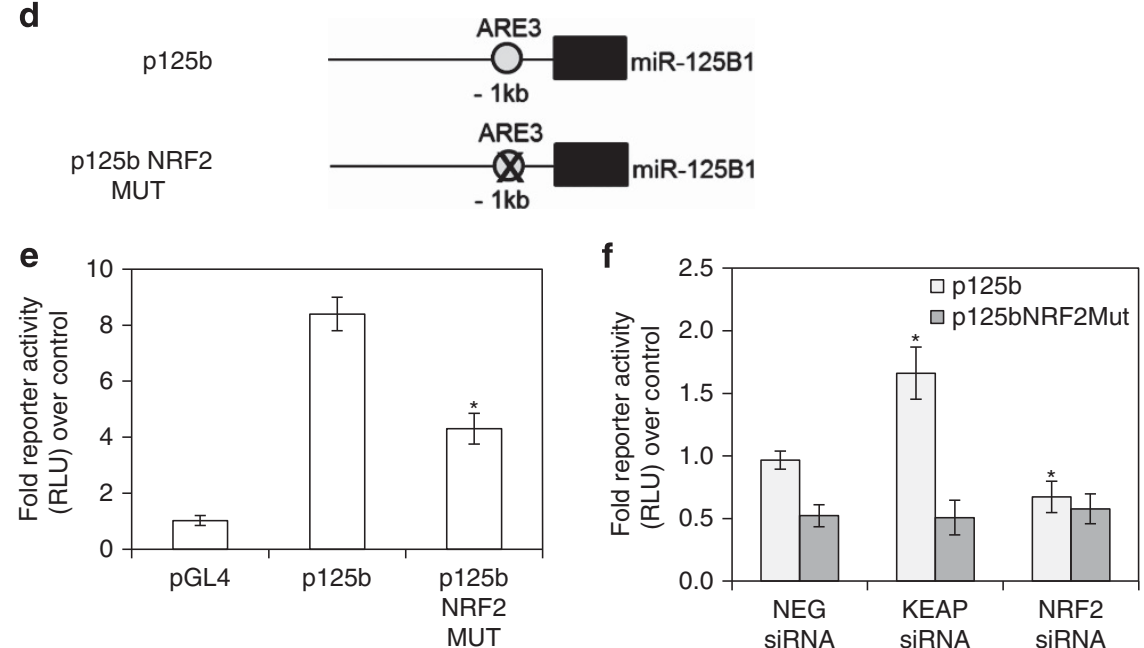

Figure 3 NRF2 binds to ARE sites in the promoters of miR-125B1 and miR-29B1. (a) Schematic presentation of ARE binding sequences in the $5^{\prime}$ region of miR-29B1 on chromosome 7 and miR-125B1 on chromosome 11. (b) Chromatin immunoprecipitation (ChIP) analysis of the miR-29B1 and miR-125B1 promoter using antibodies against NRF2 and normal rabbit lgG was used as a control. QRT-PCR was performed in triplicate on immunoprecipitated DNA and input DNA. Data presented as percent of input. ${ }^{*}$ indicates $P<0.05$ between the different treatment groups. (c) THP-1 cells were transfected with control siRNA and KEAP1 siRNA for $24 \mathrm{~h}$ and ChIP was performed. Real-time PCR was performed in triplicate on immunoprecipitated DNA and input DNA. Data presented as percent of input. * indicates $P<0.05$ between the different treatment groups. (d) Schematic representation of mutated miR-125B promoter sequence. (e) THP-1 cells were transiently transfected with $0.5 \mu \mathrm{g}$ of each promoter construct including control plasmid and pRL-TK for normalisation of transfection efficiency. Cell extracts were harvested and luciferase assays were performed. Values are the means \pm S.D., $n=4$. * indicates $P<0.01$ of deleted ARE against PGL4 control. (f) Control, KEAP1 and NRF2 siRNA were transfected at the same time as p125b and p125bNRF2 MUT and incubated for $48 \mathrm{~h}$. Cell extracts were harvested and luciferase assays were performed. Values are the means \pm S.D., $n=4$. ${ }^{*}$ indicates $P<0.01$ of KEAP1 siRNA and NRF2 siRNA against NEG siRNA control

miR-29B in AML. Supplementary Figure 2 shows that total miR-125B is increased in AML compared with normal CD34+ HSC, whereas miR-29B is decreased in AML compared with normal CD34+ HSC. Furthermore, there is a positive correlation between NRF2 RNA expression and mir-125B1 RNA expression, with an inverse correlation between NRF2 RNA expression and miR-29B1 RNA expression in primary AML cells (Figure 4b). To confirm that NRF2 regulates miR-125B1 and miR-29B1 expression in primary human AML cells, we used NRF2 siRNA to silence NRF2 RNA expression in eight AML patient samples. NRF2 mRNA is significantly knocked down in seven out of the eight AML samples of which the majority also had significantly lower miR-125B1 expression (Figure 4c). Moreover, NRF2 siRNA in the AML samples increased miR-29B1 expression (Figure 4c). Together, these results show that knockdown of NRF2 regulates the expression of miR-125B1 and miR-29B1 in primary human AML.

miR-125B antagomiR and miR-29B mimic increases AML apoptosis and sensitivity to frontline chemotherapy agents. Previous studies in AML have suggested that the overexpression of miR-125B plays an oncogenic role by repressing apoptosis and the reduction of $\mathrm{miR}-29 \mathrm{~B}$ increases 

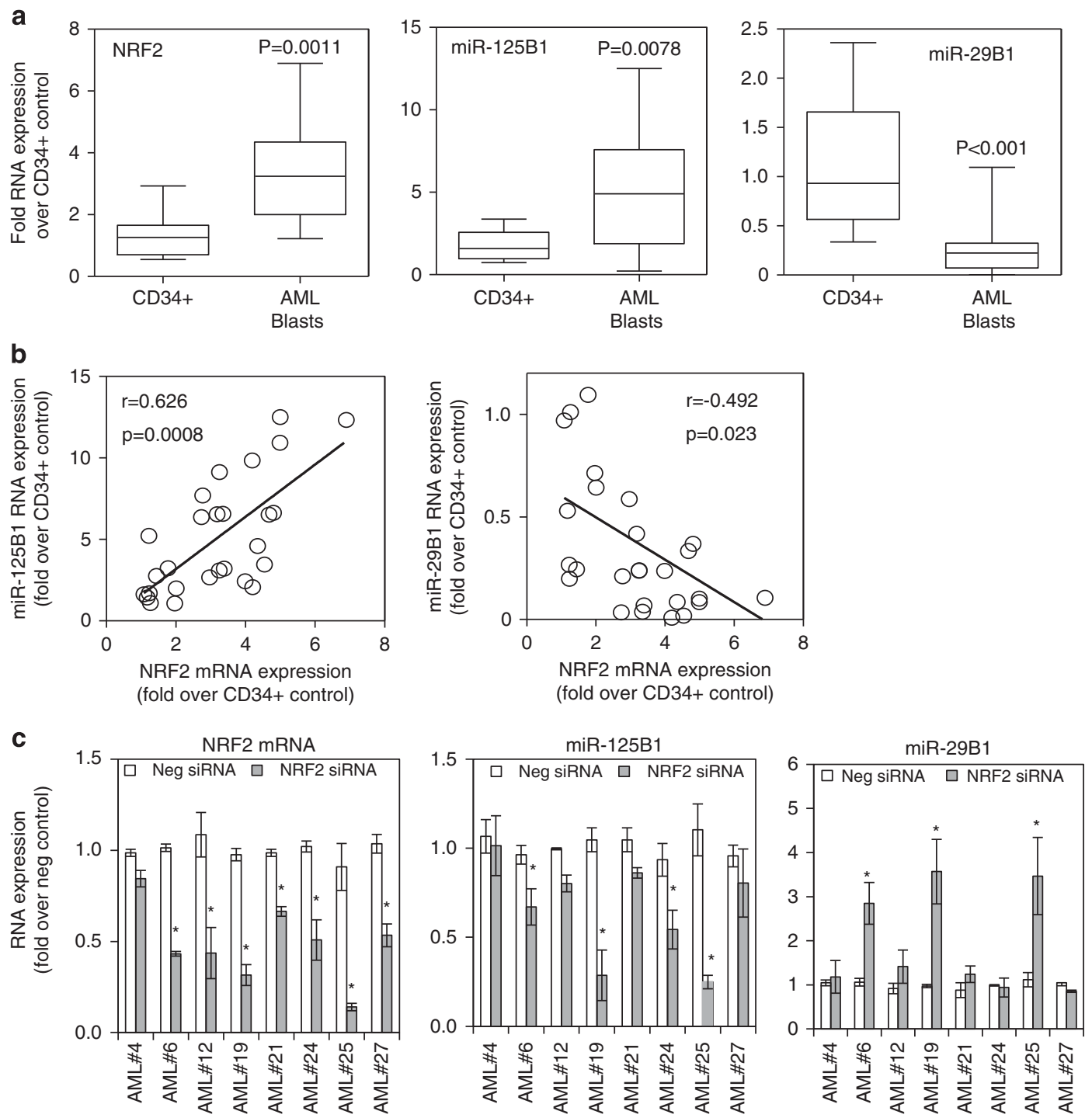

Figure 4 NRF2 regulates miR-29B1 and miR-125B1 in primary AML. (a) Total RNA was extracted from patient AML blasts $(n=18)$ and CD34+ HSC $(n=8)$. NRF2, miR-29B1 and miR-125B1 RNA expression levels were measured using QRT-PCR. (b) Pearson's correlation analysis between miR-125B1 or miR-29B1 and NRF2 mRNA expression in human primary AML cells. (c) Control and NRF2 siRNA were transfected into primary blasts $(n=7)$ for $48 \mathrm{~h}$ and RNA extracted. RNA was analysed for NRF2, miR-125B1 and miR-29B RNA expression. Values are the means \pm S.D., $n=3$

proliferation and represses apoptosis. ${ }^{18,19}$ To determine the functional role of inhibiting miR-125B and overexpressing miR-29B both alone and in-combination, we transfected miR-125B antagomiR and miR-29B mimic into THP-1 cells and analysed cells for apoptosis by annexin V/PI staining. However, before we did this, we showed that the concentrations of transfected miR-125B antagomiR and miR-29B mimic used manipulated the miRNA levels comparable with that observed in the NRF2-KD and KEAP1-KD THP-1 cells (Supplementary Figure 1). THP-1 cells transfected with the miR-125B antagomiR or miR-29B mimic showed a small but significant increase in annexin V/PI staining in comparison with the control transfection (Figure 5a). Moreover, when we co-transfected miR-125B antagomir and miR-29B mimic, we observed a synergistic increase in annexin V/PI staining in comparison with the control (Figure 5a). Transfection of
miR-125B antagomir and miR29B mimic into THP-1 and Kasumi- 1 cells potentiated the cytotoxic effect of the frontline AML chemotherapy agent daunorubicin (Supplementary Table 2).

Next, we examined the effects of inhibiting miR-125B and increasing miR-29B expression in primary $A M L$ cells in comparison with non-malignant primary CD34+ HSC. In six primary $A M L$ patient samples, inhibition of miR-125B or increasing miR-29B showed a significant decrease in cell viability (Figure $5 \mathrm{~b}$ ). Finally, we compared the viability of primary AML patient cells that had normal NRF2 expression (and thus normal miR-125B and miR-29B expression) with an AML patient sample that had high NRF2 expression (and thus high miR-125B and low miR-29B expression) after treatment with daunorubicin (Figure $5 \mathrm{c}$ and Table 1 and Supplementary Table 2). AML with normal NRF2 and normal miR-125B and 

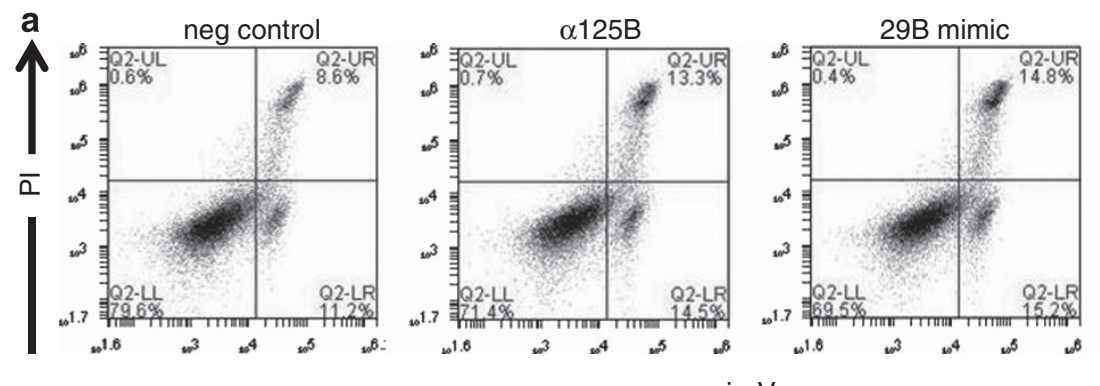

$\alpha 125 B+29 B$ mimic

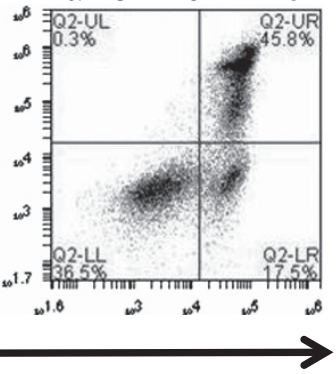

b
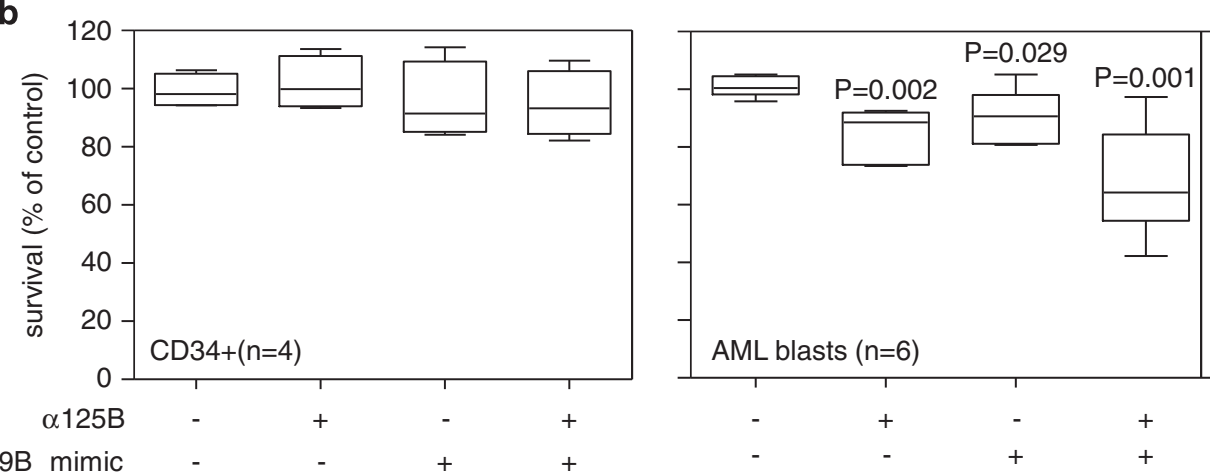

C

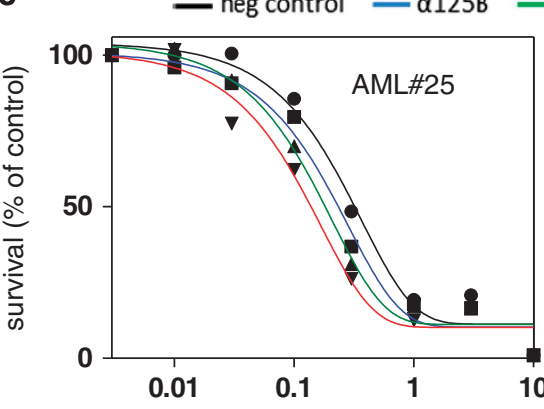

$-29 B$ mimic $-\alpha 125 B+29 B$ mimic

d
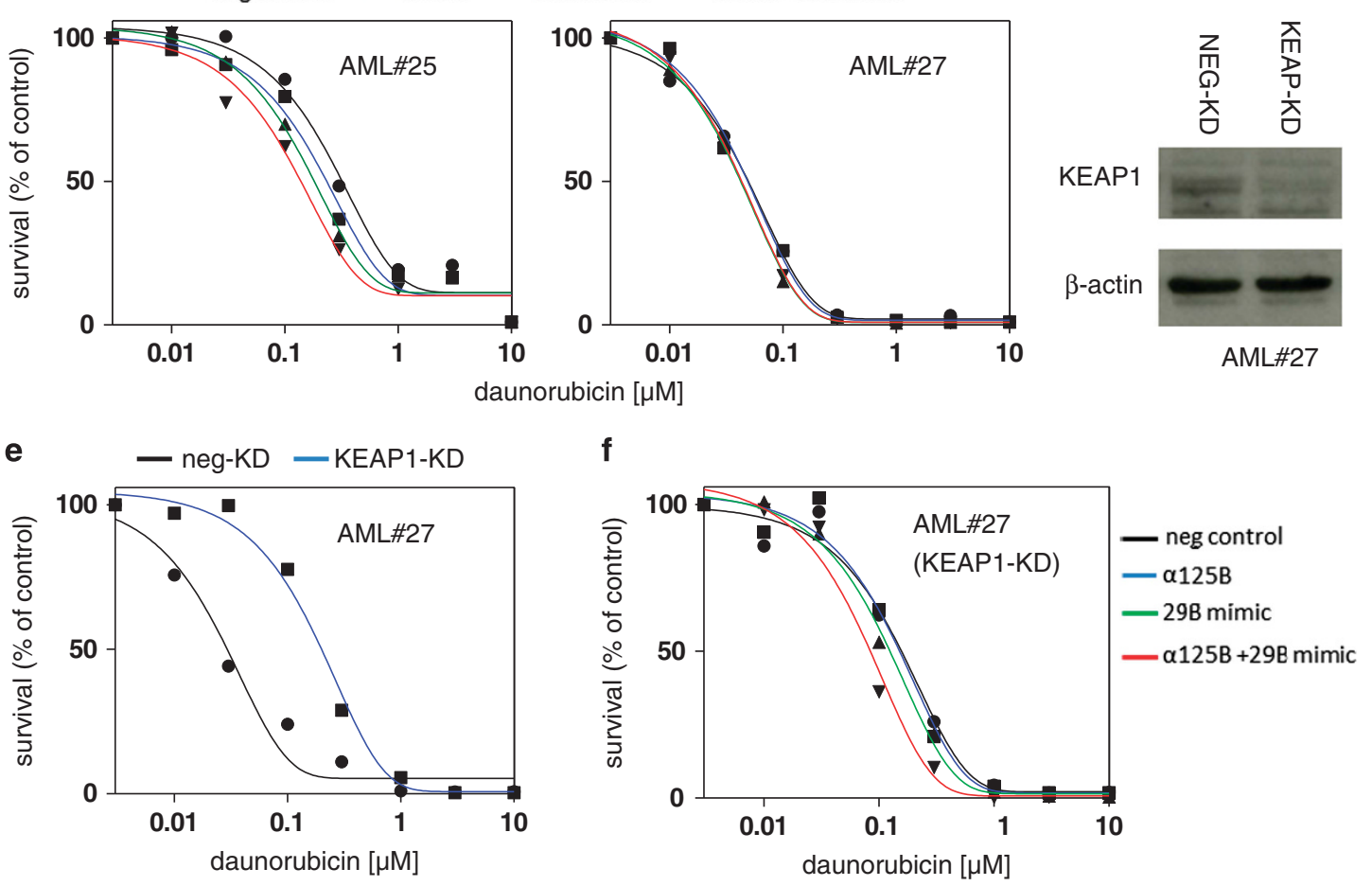

Figure 5 miR-125B antagomiR and miR-29B mimic induce apoptosis of AML cells and increases there sensitivity to AML chemotherapy. (a) THP-1 were transfected with control miRNA, miR-125B antagomiR ( $\alpha 125 \mathrm{~B})$, miR-29B mimic (29B mimic) and miR-125B antagomiR in combination with miR-29B mimic for $48 \mathrm{~h}$ before cells were analysed for apoptosis by PI/Annexin V staining. (b) AML blasts $(n=6)$ and CD34+ $(n=4)$ were transfected with control miRNA, miR-125B antagomiR and miR-29B mimic and miR-125B antagomiR in combination with miR-29B mimic for $24 \mathrm{~h}$ and then assessed for cell viability by Cell Titer-GLO. (c) AML blasts and CD34+ cells were transfected with control miRNA (miR-mimic negative control and the anti-miR miRNA inhibitor negative control for single experiments and combined miR-mimic negative control and the anti-miR miRNA inhibitor negative control in combined experiments), miR-125B antagomiR, miR-29B mimic and miR-125B antagomiR in combination with miR-29B mimic for $24 \mathrm{~h}$ before the addition of increasing doses of daunorubicin for $48 \mathrm{~h}$. Cells assessed for viability by Cell Titer-GLO. (d) AML\#27 was transduced with NEG-KD and KEAP1-KD for $48 \mathrm{~h}$ before cells before the addition of increasing doses of daunorubicin for $48 \mathrm{~h}$ before cells were analysed for KEAP1 using western blotting. Blots were reprobed for $\beta$-actin to show sample loading. (e) AML\#27 was transduced with NEG-KD and KEAP1-KD for $48 \mathrm{~h}$ before the addition of increasing doses of daunorubicin for $48 \mathrm{~h}$. Cells were assessed for viability by Cell Titer-GLO. (f) AML\#27 was transduced with KEAP1-KD for $48 \mathrm{~h}$ before cells were transfected with control miRNA, miR-125B antagomiR, miR-29B mimic and miR-125B antagomiR in combination with miR-29B mimic for $24 \mathrm{~h}$ followed by the addition of increasing doses of daunorubicin for $48 \mathrm{~h}$. Cells were assessed for viability by Cell Titer-GLO 
Table 1 AML patient sample information, NRF2 RNA expression and relative miR-125B1 and miR-29B1 RNA expression levels

\begin{tabular}{|c|c|c|c|c|c|c|c|c|}
\hline \multirow[t]{2}{*}{ Number } & \multirow[t]{2}{*}{ Age } & \multirow[t]{2}{*}{ Gender } & \multirow[t]{2}{*}{ WHO diagnosis } & \multirow[t]{2}{*}{ Cytogenetics } & \multirow[t]{2}{*}{$\%$ Blasts } & \multirow{2}{*}{$\begin{array}{l}\text { Fold NRF2 mRNA } \\
\text { over CD34+ }\end{array}$} & \multicolumn{2}{|c|}{ Fold RNA over CD34+ } \\
\hline & & & & & & & miR-125B1 & miR-29B1 \\
\hline AML\#1 & 49 & $\mathrm{M}$ & AML with maturation & Normal & 80 & 3.35 & 6.57 & 0.04 \\
\hline AML\#2 & 39 & $\mathrm{M}$ & AML with maturation & Normal & $65^{*}$ & 6.89 & 12.33 & 0.11 \\
\hline AML\#3 & 64 & $\mathrm{M}$ & AML with RUNX1-RUNX1T1 & $t(8 ; 21)$ & 85 & 1.26 & 1.09 & 1.01 \\
\hline AML\#4 & 92 & $\mathrm{~F}$ & AML with myelodysplasia related changes & Not available & $70^{*}$ & 1.23 & 1.70 & 0.20 \\
\hline AML\#5 & 82 & $\mathrm{~F}$ & AML with MDSrelated changes & Deletion 13 & 85 & 3.39 & 3.20 & 0.07 \\
\hline AML\#6 & 46 & $\mathrm{~F}$ & AML with maturation & $+4,+8, \mathrm{t}(9 ; 22)$ & $70^{*}$ & 4.99 & 12.51 & 0.10 \\
\hline AML\#7 & 66 & $\mathrm{~F}$ & AML with maturation & $\mathrm{t}(2 ; 12)$ & $65^{\star}$ & 1.23 & 5.22 & 0.27 \\
\hline AML\#8 & 78 & $\mathrm{M}$ & AML with MDS related changes & Not available & 85 & 4.19 & 9.85 & 0.01 \\
\hline AML\#9 & 57 & $\mathrm{M}$ & AML without maturation & Not available & 95 & 4.35 & 4.59 & 0.09 \\
\hline AML\#10 & 27 & $\mathrm{M}$ & AML with RUNX1-RUNX1T1 & $t(8 ; 21)$ & $60^{*}$ & 4.99 & 10.93 & 0.08 \\
\hline AML\#11 & 25 & $\mathrm{M}$ & AML with maturation & Normal & $50^{*}$ & 4.81 & 6.63 & 0.37 \\
\hline AML\#12 & 61 & $\mathrm{M}$ & Relapsed AML without maturation & Not available & 95 & 1.96 & 1.07 & 0.71 \\
\hline AML\#13 & 28 & $\mathrm{~F}$ & Acute Monoblastic and Monocytic Leukaemia & Normal & 90 & 4.68 & 6.52 & 0.33 \\
\hline AML\#14 & 31 & $\mathrm{~F}$ & AML without maturation & Trisomy 8 & $75^{*}$ & 2.73 & 6.37 & 0.03 \\
\hline AML\#15 & 84 & M & Acute meyloid leukaemia, NOS & Not available & $70^{*}$ & 3.99 & 2.43 & 0.24 \\
\hline AML\#16 & 53 & $\mathrm{M}$ & AML with $\mathrm{t}(6 ; 9)(\mathrm{p} 23 ; \mathrm{q} 34) ; D E K-N U P 214$ & $\mathrm{t}(6 ; 9)$ & $65^{*}$ & 3.25 & 9.13 & 0.24 \\
\hline AML\#17 & 51 & $\mathrm{~F}$ & AML with maturation & Normal & $40^{*}$ & 4.54 & 3.45 & 0.02 \\
\hline AML\#18 & 47 & $\mathrm{M}$ & Acute myeloid leukaemia without maturation & Not available & 90 & 4.20 & 2.07 & 1.33 \\
\hline AML\#19 & 77 & $\mathrm{~F}$ & AML with maturation & Normal & $70^{*}$ & 2.78 & 7.70 & 0.21 \\
\hline AML\#20 & 62 & $\mathrm{M}$ & AML with maturation & Complex & $55^{*}$ & 2.96 & 2.67 & 0.59 \\
\hline AML\#21 & 70 & $\mathrm{M}$ & AML with minimal differentiation & Normal & 95 & 2.01 & 1.98 & 0.64 \\
\hline AML\#22 & 65 & $\mathrm{~F}$ & AML with maturation & Normal & $40^{*}$ & 1.44 & 2.75 & 0.24 \\
\hline AML\#23 & 77 & M & Therapy related AML & Complex & $70^{*}$ & 3.24 & 3.10 & 0.24 \\
\hline AML\#24 & 40 & $\mathrm{~F}$ & AML with minimal differentiation & Normal & 90 & 1.77 & 3.23 & 1.09 \\
\hline AML\#25 & 70 & $\mathrm{M}$ & AML without maturation & Complex & 95 & 3.18 & 6.54 & 0.42 \\
\hline AML\#26 & 91 & $\mathrm{~F}$ & AML NOS & Not available & $75^{*}$ & 1.08 & 1.63 & 0.97 \\
\hline AML\#27 & 59 & $\mathrm{~F}$ & AML with t(8;21)(q22;q22); RUNX1-RUNX1T1 & $\mathrm{t}(8 ; 21)$ & 85 & 1.17 & 1.44 & 0.53 \\
\hline
\end{tabular}

This table defines the nature of the AML disease including WHO diagnosis and cytogenetics. NRF2, miR125B1 and miR-29B1 RNA expression levels over CD34+ control cells

miR-29B expression were much more sensitive to daunorubicin than AML patient samples that had high NRF2 expression and thus high miR-125B and low miR-29B expression. Moreover, when we transfected the primary $A M L$ that had high NRF2 and high mir-125B and low miR29B with miR-125B antagomiR together with miR-29B mimic, the result was that sensitivity of these AML cells to daunorubicin was increased to a level comparable with AML with normal NRF2 expression. Because AML\#27 has low NRF2 expression over normal CD34+ HSC (Table 1), which is more sensitive to daunorubicin (Figure 5c), and miR-125B antagomiR together with miR-29B mimic had no effect on resistance to daunorubicin (Figure 5c), we examined the effect of KEAP1-KD on AML\#27 to determine if we could increase the activity of NRF2 and increase the chemoresistance of this sample. Figure $5 \mathrm{~d}$ shows KEAP1-KD in AML\#27. Figure 5 e shows that KEAP1-KD $A M L \# 27$ is more resistant to daunorubicin compared with NEG-KD. However, when we transfected AML\#27 KEAP1-KD cells with control miRNA, miR-125B antagomiR, miR-29B mimic and miR-125B antagomiR in combination with miR-29B mimic, only the combination decreased resistance to daunorubicin (Figure 5f). These results demonstrate that inhibiting miR-125B and overexpressing miR-29B in combination increases apoptosis of primary $\mathrm{AML}$ and also increases sensitivity of AML cells to current frontline chemotherapy.

miR-29B and miR-125B gene targets in human AML. Studies have shown that a number of different targets exist for both miR-29B and miR-125B in AML (Supplementary Table 3). ${ }^{18,20-28}$ Here we examine the expression of genes listed in Supplementary Table 3 for their response to transfection of control miRNA, miR-125B antagomiR, miR-29B mimic and miR-125B antagomiR in combination with miR-29B mimic. Figure 6a shows that MYBL2, AKT2, CDK4 and SP1 are inhibited by miR-29B mimic in THP-1 cells; we also observe that AKT2 and CDK4 are further inhibited by the addition of miR-125B antagomiR. From the miR-125B targets, Figure 6a shows that MAPK14, STAT3 and BAK1 show an increase in mRNA in response to miR-125B antagomiR. Next, we examined the protein expression of AKT2, STAT3 and BAK1. Figure $6 \mathrm{~b}$ shows a reduction in AKT2 protein expression in response to miR-29B mimic and miR-125B antagomiR in combination with miR-29B mimic. STAT3 showed a slight increase in response to miR-29B mimic and miR-125B antagomiR in combination with miR-29B mimic. BAK1 showed an increase in response to miR-125B antagomir and miR-125B antagomiR in combination with miR-29B mimic.

Finally, we wanted to determine whether NRF2 deregulation in AML affects miRNA levels to such an extent that expression of their target genes is changed as a consequence. Figure $6 \mathrm{c}$ shows a change in expression of AKT2, STAT3 and BAK1 but not MCL1 mRNA in response to NRF2-KD or KEAP1-KD compared with NEG-KD. Figure 6d shows changes in AKT2 and BAK1 protein expression in response to NRF2-KD and an increase in response to KEAP1-KD. Together, these results link the two major observations of this study; first, that NRF2 
a

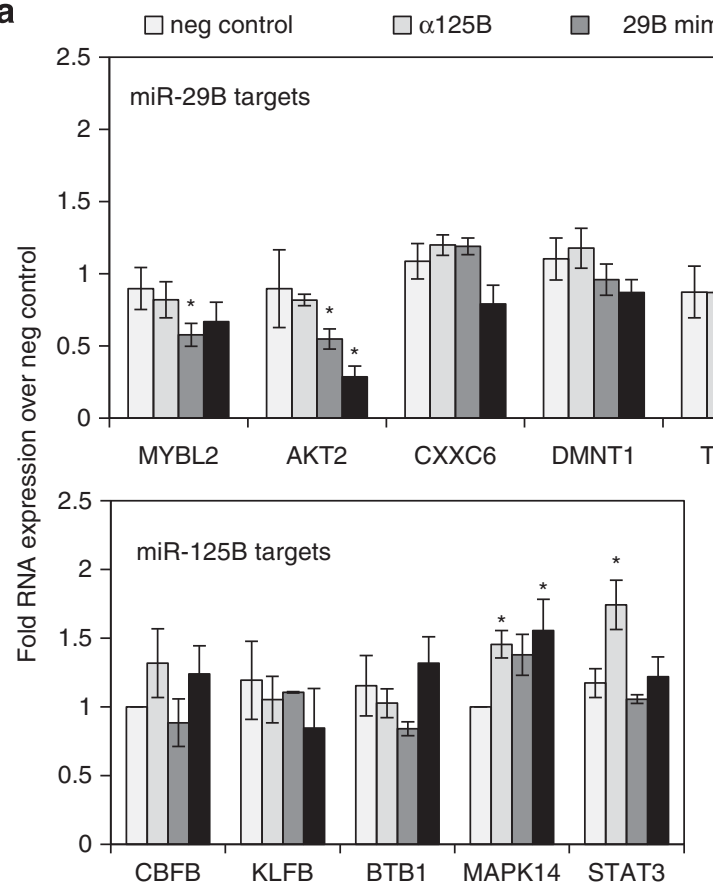

- $\alpha 125 B+29 B$ mimic
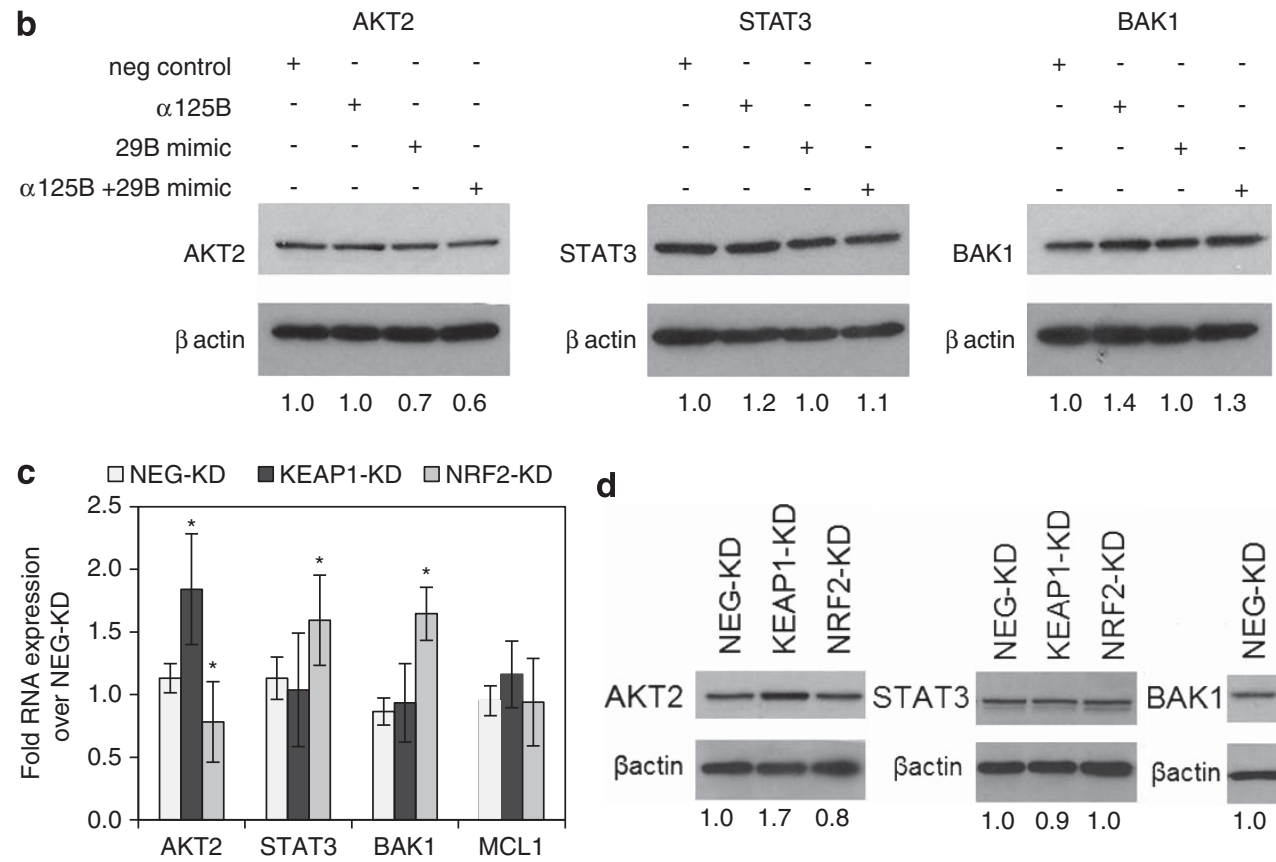

d

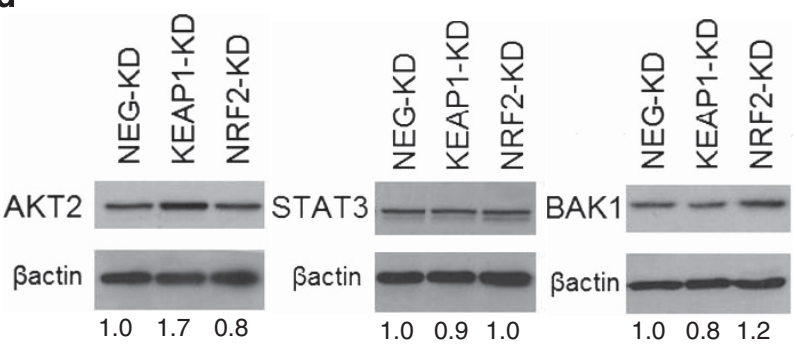

Figure 6 miR-125B antagomiR and miR-29B mimic gene targets in AML cells. (a) THP-1 were transfected with control miRNA, miR-125B antagomiR ( $\alpha 125 B)$, miR-29B mimic (29B mimic) and miR-125B antagomiR in combination with miR-29B mimic for $48 \mathrm{~h}$ before cells were analysed for target gene expression using QRT-PCR. (b) THP-1 cells were transfected with control miRNA, miR-125B antagomiR ( $\alpha 125 \mathrm{~B})$, miR-29B mimic (29B mimic) and miR-125B antagomiR in combination with miR-29B mimic for 48 $\mathrm{h}$ before cells were analysed for AKT2, STAT3 and BAK1 using western blotting. Blots were reprobed for $\beta$-actin to show sample loading. (c) QRT-PCR of mRNA for AKT2, STAT3, BAK1 and MCL1 in THP-1 cells transduced with NEG-KD, NRF2-KD or KEAP1-KD. Values represent fold change in RNA expression over NEG-KD control. (d) THP-1 cells were transduced with NEG-KD, NRF2-KD or KEAP1-KD before cells were analysed for AKT2, STAT3 and BAK1 protein expression using western blotting. Blots were reprobed for $\beta$-actin to show sample loading. The numbers under the blots indicate densitometry analysis of the blots using Image $\mathrm{J}$ software, and the results are expressed as fold change relative to the negative control

directly regulates $\mathrm{miR}-125 \mathrm{~B}$ and $\mathrm{miR}-29 \mathrm{~B}$ expression. Second, that miR-125B and miR-29B synergistically influence AML survival and chemoresistance, possibly via deregulation of target genes such as AKT2, STAT3 or BAK1.

\section{Discussion}

Our understanding of the role of non-coding RNA molecules in diseases such as cancer is presently in its infancy. To date, miRNAs have been shown to have a significant role in cancer 
biology through the regulation of both tumour suppressors and oncogenes. Less is known, however, about the upstream control of miRNA expression in cancer and the biological processes that control miRNA expression. In AML, the differential expression of a number of miRNAs has been reported to be associated with the malignant phenotype but as yet the upstream mechanisms by which this is controlled are yet to be described. ${ }^{29-32}$ As we have previously identified NRF2 as an important regulator of cell survival in AML, the aim of the present study was to investigate the ability of NRF2 to affect miRNA expression and furthermore define the function of these miRNAs in human AML both in a resting state and in response to front-line chemotherapeutic agents.

Through gene expression data and multiple transcription factor binding assays, we found that NRF2 upregulates mir-125B1 and downregulates miR-29B1 though binding to specific ARE sites in the miR-125B1 and miR-29B1 regulatory regions. Furthermore, we observed that by decreasing the expression of miR-125B and increasing the expression of miR-29B, the cells become more sensitive to apoptotic stimuli. These findings are in keeping with previous observations that miR-29B and miR-125B regulate the expression of multiple survival pathways in AML. The global repression of miR-125B targets and elevation of miR-29B targets genes would aid the uncontrolled proliferative phenotype of AML. Therefore, in Figures $6 \mathrm{a}$ and $\mathrm{b}$, we analyse the targets of miR-29B and miR-125B in AML cells transfected with corresponding mimic and antagomir, both alone and in combination. We observe changes in a number of genes including AKT2, CDK4, MAPK14, STAT3, BCL2 and BAK1. Interestingly, AKT2 and CDK4 are targets of miR-29B only; however, when we co-transfect miR-125B antagomir and miR-29B mimic, we do observe a further reduction over miR-29B mimic only. There are no miR-125B sites within these two genes suggesting that the combined observations are due to off-target effects. Together, the cumulative repression of miR-29B and the overexpression of $\mathrm{miR}-125 \mathrm{~B}$ significantly contribute to leukaemia cell proliferation within the bone marrow niche.

Transcription factors are generally structured in context of networks. In AML, miR-29B downregulation has been found to be associated with high C-MYC levels as well as high SP1 and NF- $k \mathrm{~B} .{ }^{33,34}$ Furthermore, NF- $k \mathrm{~B}$, JUN and $\mathrm{C}-\mathrm{MYC}$ have been linked to increased NRF2 activity in human cancer (including $\mathrm{AML})^{10,35}$ and NRF2 has been shown to transcriptionally regulate $\mathrm{miR}-125 \mathrm{~B}$ in kidney epithelia cells in response to cisplatin-induced toxicity. ${ }^{36}$ Taken together, it is likely that NRF2 has a central role in the complex network regulating miR-29B and miR-125B in human AML.

We also highlight that other miRNAs may be regulated by NRF2. The array data shown as Supplementary Table 1 show that some miRNAs are downregulated in response to NRF2-KD and increased in response to sulforaphane. This list includes miR-222, miR221, miR-223 miR-23a, miR-195 and miR-196a. Of particular interest from this group is miR-222, miR-221 and miR223, which have been shown to be highly expressed in AML compared with other leukaemias. ${ }^{37}$ Moreover, we also noted that high NRF2 activity caused a decrease in the expression of miRNAs, which includes miR-154, miR146a and miR-181B. All these miRNAs have been shown to be of interest in AML with miR-146a being shown to have low expression in AML, which correlates with high expression of its target gene CXCR4, ${ }^{38}$ which in a separate study has been shown to be regulated by NRF2 in human HSC. ${ }^{39}$ Consequently in human AML, NRF2 not only regulates key genes involved in the reaction to oxidative stress but also regulates miRNAs that appear to have a broader impact on gene expression and regulation.

In summary, here we report the first description of upstream miRNA regulation by NRF2 in a human cancer and its downstream cyto-protective consequences. In doing so, we not only provide novel mechanistic insight into the protumoural consequences of the constitutive NRF2 expression in $\mathrm{AML}$, but we also propose that targeting the NRF2 regulation of these miRNA (or the miRNA directly) would be a biologically plausible therapeutic strategy to increase the effectiveness of chemotherapy in human AML.

\section{Materials and Methods}

Materials. The AML-derived cell lines were obtained from the European Collection of Cell Cultures where they are authenticated by DNA fingerprinting. In the laboratory, they are used at low passage number for a maximum of 6 months post-resuscitation, testing regularly for Mycoplasma infection. All primers were purchased from Invitrogen (Paisley, UK). AKT2, STAT3 and BAK1 antibodies were purchased from Cell Signalling Technology (Cambridge, MA, USA). NRF2 and KEAP1 antibodies were purchased from Santa Cruz Biotechnology (Santa Cruz, CA, USA). All other reagents were obtained from Sigma-Aldrich (St. Louis, MO, USA), unless indicated. To assess whether NRF2 regulates miRNA expression in AML, we used commercially available OncomiR collection Cancer miRNA qRT-PCR Array (SBI, Mountain View, CA, USA).

Primary AML cell culture. AML cells were obtained from patients' bone marrow or blood following informed consent and under approval from the UK National Research Ethics Service (LRECref07/H0310/146). For primary cell isolation, heparinised blood was collected from volunteers and human peripheral blood mononuclear cells isolated by Histopaque (Sigma-Aldrich, Dorset, UK) density gradient centrifugation. AML samples that were less than $80 \%$ blasts were purified using the CD34-positive selection kit (Miltenyi Biotec, Auburn, CA, USA) (denoted by * in Table 1). Cell type was confirmed by microscopy and flow cytometry. We obtained haematopoietic CD34+ cells from two sources, Stem Cell Technologies (Manchester, UK) and volunteers. Positive selection of CD34+ cells were isolated from peripheral blood mononuclear cells using a CD34-positive selection kit (Miltenyi Biotec). For all CD34+ experiments, at least three different donors were used to obtain the results presented in this paper. Cell type was confirmed by microscopy and flow cytometry.

RNA extraction and real time PCR (RT-PCR). Total mRNA extraction was carried out using mirVana miRNA isolation kit (Ambion, Paisley, UK) and reverse transcribed using the miRscript II RT kit (Qiagen, Manchester, UK). All mature miRNA were normalised to RNU6B and immature miRNA and mRNA were normailsed to GAPDH for qRT-PCR as described previously. ${ }^{40}$ qRT-PCR was carried out using SYBR green technology (Qiagen). The samples were preamplified at $95^{\circ} \mathrm{C}$ for $2 \mathrm{~min}$, after which was amplified for 45 cycles at $95^{\circ} \mathrm{C}$ for $15 \mathrm{~s}, 60^{\circ} \mathrm{C}$ for $10 \mathrm{~s}$ and $72{ }^{\circ} \mathrm{C}$ for $10 \mathrm{~s}$. The qRT-PCR was performed on the LightCycler480 (Roche, Burgess Hill, UK).

Virus construction and infection. Lentiviruses containing miRNA sequence miRNA-Nrf2 (5'-TTAATGAGTTCACTGTCAACT-3'), miRNA-KEAP1 (5'-GTTTTGGCCACTGACTGAC- $\left.3^{\prime}\right)$ and miR-NEG were constructed and produced as described previously. ${ }^{41}$ For transduction, THP-1 cells and AML blasts cells were plated onto 12 -well plates $\left(5 \times 10^{4}\right.$ cells/well) and infected with lentiviruses (multiplicity of infection of 15) with $8 \mu \mathrm{g} / \mathrm{ml}$ Polybrene (Sigma, Dorset, UK). Transduced cells were analyzed by flow cytometry (Accuri, BD Biosciences, Oxford, UK), real-time PCR (Roche) and western blotting.

Transfections. AML transfections were carried out with $2 \times 10^{6}$ cells using the Amaxa Nucleofactor Kit II (Cambridge, UK). Control miR, miR-29B mimic and miR-125B antagomiR, control siRNA, KEAP1 and NRF2 siRNA were purchased 
from Invitrogen were transfected in at a concentration of $45 \mathrm{~nm}$. For control miR, the miR-mimic negative control and the anti-miR miRNA inhibitor negative control for single experiments and combined miR-mimic negative control and the anti-miR miRNA inhibitor negative control in combined experiments were used. For all gene expression experiments, the cells were incubated for $24 \mathrm{~h}$ post transfection before RNA extraction. For the cell viability assays, AML cells were incubated for $48 \mathrm{~h}$ post transfection. For reporter assays, $0.5 \mu \mathrm{g}$ of PGL4 reporter and pRL-TK control constructs were co-transfected into THP-1. Transfected cells were incubated for $24 \mathrm{~h}$ before the indicated treatments. For reporter assay, cells were treated with DualLuciferase reporter assay system (Promega, Southampton, UK).

Western blotting. Sodium dodecyl sulfate-polyacrylamide gel electrophoresis and western blot analyses were performed as described previously. Briefly, whole cell lysates as well as nuclear and cytosolic were extracted and sodium dodecyl sulfate-polyacrylamide gel electrophoresis separation was performed. ${ }^{42}$ Protein was transferred to nitrocellulose and western blot analysis performed with the indicated antisera according to their manufacturer's guidelines.

Cloning of miR-125B promoter construct. To generate the $\mathrm{p} 125 \mathrm{~B}$ promoter construct containing ARE3, a DNA fragment containing $2.6 \mathrm{~kb}$ of the human 125b promoter region was amplified from genomic DNA with PCR and specific primers: 5'-TGAGAGGAGCGCAACAATG-3' reverse primer and 5'-AGAAAGGCCACCAAGATTCAC-3' forward primer. The fragment was cloned into the PGL4.11 basic plasmid (Promega). To generate mutated NRF2 construct (p125B NRF2 MUT) the sense PCR primer used was 5'-GCTGTG GCTGtTTTGTATTCTCTTTGACTAG-3'. This mutation was introduced with the QuikChange XL Site-Directed Mutagenesis Kit (Agilent, Stockport, UK).

Cell viability and apoptosis assays. THP-1, Kasumi-1, primary CD34+ HSC and primary AML cells were transfected with control miR (miR-mimic negative control and the anti-miR miRNA inhibitor negative control for single experiments and combined miR-mimic negative control and the anti-miR miRNA inhibitor negative control in combined experiments), miR-29B mimic and miR-125B antagomiR and combined miR-29B mimic and miR-125B antagomiR for $24 \mathrm{~h}$. THP-1 cells were analysed for apoptosis using PI/Annexin V staining. THP-1, Kasumi-1, primary CD34+ HSC and primary AML transfected cells were treated with different concentrations of chemotherapy agents and incubated for $48 \mathrm{~h}$; then, viable cells were measured with Cell-Titre GLO (Promega).

Chromatin immunoprecipitation assay. THP-1 cells were transfected with control siRNA or KEAP1 siRNA $24 \mathrm{~h}$ before the cells were fixed with $1 \%$ formaldehyde in medium for $10 \mathrm{~min}$ at room temperature. The sonication conditions were optimised to determine generation of DNA fragments between 300 and 600 base pairs in length. Chromatin was immunoprecipitated with IgG, anti-NRF2 (Cell Signalling). The association of NRF2 was measured by RT-PCR on immunoprecipitated chromatin using primers spanning the ARE sites described in Figure $3 a$ and Supplementary Table 4.

Statistical analyses. Student's $t$ test was performed to assess statistical significance from controls. Results with $P<0.05$ were considered statistically significant $\left(^{*}\right)$. Results represent the mean \pm S.D. of three independent experiments.

\section{Conflict of Interest}

The authors declare no conflict of interest.

Acknowledgements. We wish to thank the World Cancer Research, National Institutes for Health Research (Flexibility and Sustainability Funding) and The Big C. We thank Professor Richard Ball at the Norfolk and Norwich University Hospital tissue bank for assistance with primary tissue collection.

1. Estey E, Dohner H. Acute myeloid leukaemia. Lancet 2006; 368: 1894-1907.

2. Kvinlaug BT, Chan WI, Bullinger L, Ramaswami M, Sears C, Foster D et al. Common and overlapping oncogenic pathways contribute to the evolution of acute myeloid leukemias. Cancer Res 2011; 71: 4117-4129.

3. Moi P, Chan K, Asunis I, Cao A, Kan YW. Isolation of NF-E2-related factor 2 (Nrf2), a NF-E2like basic leucine zipper transcriptional activator that binds to the tandem NF-E2/AP1 repeat of the beta-globin locus control region. Proc Natl Acad Sci USA 1994; 91: 9926-9930.
4. Alam J, Stewart D, Touchard C, Boinapally S, Choi AMK, Cook JL. Nrf2, a Cap'n'Collar transcription factor, regulates induction of the heme oxygenase-1 gene. J Biol Chem 1999; 274: 26071-26078.

5. Venugopal R, Jaiswal AK. Nrf1 and Nrf2 positively and c-Fos and Fra1 negatively regulate the human antioxidant response element-mediated expression of $N A D(P) H: q u i n o n e$ oxidoreductase1 gene. Proc Natl Acad Sci USA 1996; 93: 14960-14965.

6. Ooi A, Dykema K, Ansari A, Petillo D, Snider J, Kahnoski R et al. CUL3 and NRF2 mutations confer an NRF2 activation phenotype in a sporadic form of papillary renal cell carcinoma. Cancer Res 2013; 73: 2044-2051.

7. Hanada N, Takahata T, Zhou Q, Ye X, Sun R, Itoh J et al. Methylation of the KEAP1 gene promoter region in human colorectal cancer. BMC Cancer 2012; 12: 66

8. Kim YR, Oh JE, Kim MS, Kang MR, Park SW, Han JY et al. Oncogenic NRF2 mutations in squamous cell carcinomas of oesophagus and skin. $J$ Pathol 2011; 220: 446-451.

9. Konstantinopoulos PA, Spentzos D, Fountzilas E, Francoeur N, Sanisetty S, Grammatikos AP et al. Keap1 mutations and Nrf2 pathway activation in epithelial ovarian cancer. Cancer Res 2011; 71: 5081-5089.

10. Rushworth SA, Zaitseva L, Murray MY, Shah NM, Bowles KM, MacEwan DJ. The high Nrf2 expression in human acute myeloid leukemia is driven by NF- $\mathrm{\kappa B}$ and underlies its chemoresistance. Blood 2012; 120: 5188-5198.

11. Sporn MB, Liby KT. NRF2 and cancer: the good, the bad and the importance of context. Nat Rev Cancer 2012; 12: 564-571.

12. Rushworth SA, Bowles KM, MacEwan DJ. High basal nuclear levels of Nrf2 in acute myeloid leukemia reduces sensitivity to proteasome inhibitors. Cancer Res 2011; 71: 1999-2009.

13. Winter J, Jung S, Keller S, Gregory RI, Diederichs S. Many roads to maturity: microRNA biogenesis pathways and their regulation. Nat Cell Biol 2009; 11: 228-234.

14. Garzon R, Marcucci G, Croce CM. Targeting microRNAs in cancer: rationale, strategies and challenges. Nat Rev Drug Discovery 2010; 9: 775-789.

15. Chorley BN, Campbell MR, Wang X, Karaca M, Sambandan D, Bangura F et al. Identification of novel NRF2-regulated genes by ChIP-Seq: influence on retinoid $X$ receptor alpha. Nucleic Acids Res 2012; 40: 7416-7429.

16. Devling TWP, Lindsay CD, McLellan LI, McMahon M, Hayes JD. Utility of siRNA against Keap1 as a strategy to stimulate a cancer chemopreventive phenotype. Proc Natl Acad Sci USA 2005; 102: 7280-7285.

17. Zhao $\mathrm{CR}$, Gao ZH, Qu XJ. Nrf2-ARE signaling pathway and natural products for cancer chemoprevention. Cancer Epidemiol 2010; 34: 523-533.

18. Garzon R, Heaphy CE, Havelange V, Fabbri M, Volinia S, Tsao T et al. MicroRNA 29b functions in acute myeloid leukemia. Blood 2009; 114: 5331-5341.

19. Bousquet M, Harris MH, Zhou B, Lodish HF. MicroRNA miR-125b causes leukemia. Proc Natl Acad Sci USA 2010; 107: 21558-21563.

20. Gong J, Zhang JP, Li B, Zeng C, You K, Chen MX et al. MicroRNA-125b promotes apoptosis by regulating the expression of Mcl-1, Bcl-w and IL-6R. Oncogene 2013; 32: 3071-3079.

21. Zhang $\mathrm{H}$, Luo $X Q$, Feng $D D$, Zhang $X J$, Wu J, Zheng YS et al. Upregulation of microRNA-125b contributes to leukemogenesis and increases drug resistance in pediatric acute promyelocytic leukemia. Mol Cancer 2011; 10: 108.

22. Garzon R, Liu S, Fabbri M, Liu Z, Heaphy CE, Callegari E et al. MicroRNA-29b induces global DNA hypomethylation and tumor suppressor gene reexpression in acute myeloid leukemia by targeting directly DNMT3A and 3B and indirectly DNMT1. Blood 2009; 113: 6411-6418.

23. Zhao JJ, Lin J, Lwin T, Yang H, Guo J, Kong W et al. microRNA expression profile and identification of miR-29 as a prognostic marker and pathogenetic factor by targeting CDK6 in mantle cell lymphoma. Blood 2010; 115: 2630-2639.

24. Bousquet M, Nguyen D, Chen C, Shields L, Lodish HF. MicroRNA-125b transforms myeloid cell lines by repressing multiple mRNA. Haematologica 2012; 97: 1713-1721.

25. Ooi AG, Sahoo D, Adorno M, Wang Y, Weissman IL, Park CY. MicroRNA-125b expands hematopoietic stem cells and enriches for the lymphoid-balanced and lymphoid-biased subsets. Proc Natl Acad Sci USA 2010; 107: 21505-21510.

26. Tan G, Niu J, Shi Y, Ouyang H, Wu ZH. NF-kappaB-dependent microRNA-125b up-regulation promotes cell survival by targeting p38alpha upon ultraviolet radiation. J Biol Chem 2012; 287: 33036-33047.

27. Surdziel E, Cabanski M, Dallmann I, Lyszkiewicz M, Krueger A, Ganser A et al. Enforced expression of miR-125b affects myelopoiesis by targeting multiple signaling pathways. Blood 2011; 117: 4338-4348.

28. Luna C, Li G, Qiu J, Epstein DL, Gonzalez P. Cross-talk between miR-29 and transforming growth factor-betas in trabecular meshwork cells. Invest Ophthalmol Vis Sci 2011; 52: 3567-3572.

29. Anderson JE, Kopecky KJ, Willman CL, Head D, O'Donnell MR, Luthardt FW et al. Outcome after induction chemotherapy for older patients with acute myeloid leukemia is not improved with mitoxantrone and etoposide compared to cytarabine and daunorubicin: a Southwest Oncology Group study. Blood 2002; 100: 3869-3876.

30. Wang H, Tan G, Dong L, Cheng L, Li K, Wang Z et al. Circulating MiR-125b as a Marker Predicting Chemoresistance in Breast Cancer. PLoS One 2012; 7: e34210.

31. Okamoto K, Miyoshi K, Murawaki Y. miR-29b, miR-205 and miR-221 Enhance Chemosensitivity to Gemcitabine in HuH28 Human Cholangiocarcinoma Cells. PLoS One 2013; 8: e77623. 
32. Blum W, Garzon R, Klisovic RB, Schwind S, Walker A, Geyer S et al. Clinical response and miR-29b predictive significance in older AML patients treated with a 10-day schedule of decitabine. Proc Natl Acad Sci USA 2010; 107: 7473-7478.

33. Liu S, Wu LC, Pang J, Santhanam R, Schwind S, Wu YZ et al. Sp1/NFkappaB/HDAC/ miR-29b regulatory network in KIT-driven myeloid leukemia. Cancer Cell 2010; 17: 333-347.

34. Gong JN, Yu J, Lin HS, Zhang XH, Yin XL, Xiao Z et al. The role, mechanism and potentially therapeutic application of microRNA-29 family in acute myeloid leukemia. Cell Death Differ 2014; 21: 100-112.

35. DeNicola GM, Karreth FA, Humpton TJ, Gopinathan A, Wei C, Frese K et al. Oncogeneinduced Nrf2 transcription promotes ROS detoxification and tumorigenesis. Nature 2011; 475: 106-109.

36. Kong F, Sun C, Wang Z, Han L, Weng D, Lu Y et al. miR-125b confers resistance of ovarian cancer cells to cisplatin by targeting pro-apoptotic Bcl-2 antagonist killer 1. J Huazhong Univ Sci Technolog Med Sci 2011; 31: 543-549.
37. Wang $Y$, Li Z, He C, Wang D, Yuan X, Chen $\mathrm{J}$ et al. MicroRNAs expression signatures are associated with lineage and survival in acute leukemias. Blood Cells Mol Dis 2010; 44: 191-197.

38. Spinello I, Quaranta MT, Riccioni R, Riti V, Pasquini L, Boe A et al. MicroRNA-146a and AMD3100, two ways to control CXCR4 expression in acute myeloid leukemias. Blood Cancer J 2011; 1: e26.

39. Tsai JJ, Dudakov JA, Takahashi K, Shieh J-H, Velardi E, Holland AM et al. Nrf2 regulates haematopoietic stem cell function. Nat Cell Biol 2013; 15: 309-316.

40. Murray MY, Rushworth SA, Zaitseva L, Bowles KM, MacEwan DJ. Attenuation of dexamethasone-induced cell death in multiple myeloma is mediated by miR-125b expression. Cell Cycle 2013; 12: 2144-2153.

41. Zaitseva L, Rushworth SA, MacEwan DJ. Silencing FLIPL modifies TNF-induced apoptotic protein expression. Cell Cycle 2011; 10: 1067-72.

42. Rushworth SA, MacEwan DJ. HO-1 underlies resistance of AML cells to TNF-induced apoptosis. Blood 2008; 111: 3793-3801.

Supplementary Information accompanies this paper on Cell Death and Differentiation website (http://www.nature.com/cdd) 